\title{
HADRONS IN THE NUCLEAR MEDIUM ${ }^{1}$
}

\author{
U. MOSEL ${ }^{2}$ \\ Institut fuer Theoretische Physik, Universitaet Giessen \\ D-35392 Giessen, Germany
}

\begin{abstract}
In this talk I first discuss predictions based on QCD sum rules and on hadronic models for the properties of vector mesons in the nuclear medium. I then describe possible experimental signatures and show detailed predictions for dilepton invariant mass spectra with a special emphasis on nuclear reactions involving elementary incoming beams and nuclear targets. I will in particular illustrate that the sensitivity of pion and photon induced reactions to in-medium effects is nearly as large as that of heavy-ion reactions.
\end{abstract}

\section{そ1 Introduction}

The investigation of so-called in-medium properties of hadrons has found widespread interest during the last decade. This interest was triggered by two aspects.

The first aspect that triggered an interest in the investigation of in-medium properties of hadrons was a QCD sum-rule based prediction by Hatsuda and Lee [1] in 1992 that the masses of vector mesons should drop dramatically as a function of nuclear density. It was widely felt that an experimental everification of this prediction would establish a long-sought direct link between quark degrees of freedom and nuclear hadronic interactions. In the same category fall the predictions of Brown and Rho that argued for a general scaling for hadron masses with density [2].

The second aspect is that even in ultrarelativistic heavy-ion reactions, searching for observables of a quark-gluon plasma phase of nuclear matter, inevitably also many relatively low-energy $(\sqrt{s} \approx$ $2-4 \mathrm{GeV}$ ) final state interactions take place. These interactions involve, for example, collisions between many mesons for which the cross-sections and meson self-energies in the nuclear medium are not known, but may influence the interpretation of the experimental results.

Hadron properties in medium involve masses, widths and coupling strengths of these hadrons. In lowest order in the nuclear density all of these are linked by the $t \rho$ approximation that assumes that the interaction of a hadron with many nucleons is simply given by the elementary $t$-matrix of the hadron-nucleon interaction multiplied with the nuclear density $\rho$. For vector mesons this approximation reads

$$
\Pi_{\mathrm{V}}=-4 \pi f_{\mathrm{VN}}(0) \rho
$$

\footnotetext{
${ }^{1}$ work supported by BMBF and DFG

${ }^{2}$ mosel@theo.physik.uni-giessen.de
} 
where $f_{\mathrm{VN}}$ is the forward-scattering amplitude of the vector meson $(\mathrm{V})$ nucleon $(\mathrm{N})$ interaction. Approximation (11) is good for low densities $\left(\Pi_{\mathrm{V}}\right.$ is linear in $\rho$ ) and/or large relative momenta where the vector meson 'sees' only one nucleon at a time. Relation (11) also neglects the Fermi-motion of the nucleons although this could easily be included.

Simple collision theory [3, 团 then gives the shift of mass and width of a meson in nuclear matter as

$$
\begin{aligned}
\delta m_{\mathrm{V}} & =-\gamma v \sigma_{\mathrm{VN}} \eta \rho \\
\delta \Gamma_{\mathrm{V}} & =\gamma v \sigma_{\mathrm{VN}} \rho .
\end{aligned}
$$

Here, according to the optical theorem, $\eta$ is given by the ratio of real to imaginary part of the forward scattering amplitude

$$
\eta=\frac{\Re f_{\mathrm{VN}}(0)}{\Im f_{\mathrm{VN}}(0)} .
$$

The expressions (2) are interesting since an experimental observation of these mass- and widthchanges could give valuable information on the free cross sections $\sigma_{\mathrm{VN}}$ which may not be available otherwise. The more fundamental question, however, is if there is more to in-medium properties than just the simple collisional broadening predictions of (因).

\section{Fundamentals of Dilepton Production}

From QED it is well known that vacuum polarization, i.e. the virtual excitation of electron-positron pairs, can dress the photon. Because the quarks are charged, also quark-antiquark loops can dress the photon. These virtual quark-antiquark pairs have to carry the quantum numbers of the photon, i.e. $J^{\pi}=1^{-}$. The $q \bar{q}$ pairs can thus be viewed as vector mesons which have the same quantum numbers; this is the basis of Vector Meson Dominance (VMD).

The vacuum polarization tensor is then, in complete analogy to QED, given by

$$
\Pi^{\mu \nu}=\int d^{4} x e^{i q x}\left\langle 0\left|T\left[j^{\mu}(x) j^{\nu}(0)\right]\right| 0\right\rangle=\left(g^{\mu \nu}-\frac{q^{\mu} q^{\nu}}{q^{2}}\right) \Pi\left(q^{2}\right)
$$

where $T$ is the time ordering operator. Here, in the second line, the tensor structure has been exhibited explicitly. This so-called current-current correlator contains the currents $j^{\mu}$ with the correct charges of the vector mesons in question. Simple VMD [5] relates these currents to the vector meson fields

$$
j^{\mu}(x)=\frac{m_{\mathrm{V}}^{0}{ }^{2}}{g_{\mathrm{V}}} V^{\mu}(x) .
$$

Using this equation one immediately sees that the current-current correlator (困) is nothing else but the vector meson propagator $D_{\mathrm{V}}$

$$
\Pi\left(q^{2}\right)=\left(\frac{m_{\mathrm{V}}^{0}{ }^{2}}{g_{\mathrm{V}}}\right)^{2} D_{\mathrm{V}}\left(q^{2}\right)
$$


The scalar part of the vector meson propagator is given by

$$
D_{\mathrm{V}}\left(q^{2}\right)=\frac{1}{q^{2}-m_{\mathrm{V}}^{0^{2}}-\Pi_{\mathrm{V}}\left(q^{2}\right)} .
$$

Here $\Pi_{\mathrm{V}}$ is the selfenergy of the vector meson.

For the free $\rho$ meson information about $\Pi\left(q^{2}\right)$ can be obtained from hadron production in $e^{+} e^{-}$ annihilation reactions [6]

$$
R(s)=\frac{\sigma\left(e^{+} e^{-} \rightarrow \text { hadrons }\right)}{\sigma\left(e^{+} e-\rightarrow \mu^{+} \mu^{-}\right)}=-\frac{12 \pi}{s} \Im \Pi(s)
$$

with $s=q^{2}$. This determines the imaginary part of $\Pi$ and, invoking vector meson dominance, also of $\Pi_{\mathrm{V}}$. The data (see, e.g. Fig. 18.8 in [6], or Fig. 1 in [7]) clearly show at small $\sqrt{s}$ the vector meson peaks, followed by a flat plateau starting at $\sqrt{s} \approx 1.5 \mathrm{GeV}$ described by perturbative QCD.

In order to get the in-medium properties of the vector mesons, i.e. their selfenergy $\Pi_{V}$, we now have two ways to proceed: We can, first, try to determine the current-current correlator by using QCD sum rules [1]; from this correlator we can then determine the self-energy of the vector meson following eqs. (6),(7). The second approach consists in setting up a hadronic model and calculating the selfenergy of the vector meson by simply dressing its propagators with appropriate hadronic loops. In the following sections I will discuss both of these approaches.

\subsection{QCD sum rules and in-medium masses}

The QCD sum rule for the current-current correlator is obtained by evaluating the function $R(s)$, and thus $\Im \Pi(s)$ (see (8)), in a hadronic model on one hand and in a QCD-based model on the other. The latter, QCD based, calculation uses the fact that the current-current correlator (₫) can be Taylor expanded in the space-time distance $x$ for small space-like distances between $x$ and 0 ; this is nothing else than the Operator Product Expansion (OPE) [6]. In this way we obtain for the free meson

$$
R^{\mathrm{OPE}}\left(M^{2}\right)=\frac{1}{8 \pi^{2}}\left(1+\frac{\alpha_{\mathrm{S}}}{\pi}\right)+\frac{1}{M^{4}} m_{\mathrm{q}}\langle\bar{q} q\rangle+\frac{1}{24 M^{4}}\left\langle\frac{\alpha_{\mathrm{S}}}{\pi} G^{2}\right\rangle-\frac{56}{81 M^{6}} \pi \alpha_{\mathrm{S}} \kappa\langle\bar{q} q\rangle^{2} .
$$

Here $M$ denotes the so-called Borel mass. The expectation values appearing here are the quark- and gluon-condensates. The last term here contains the mean field approximation

$$
\left\langle(\bar{q} q)^{2}\right\rangle=\kappa\langle\bar{q} q\rangle^{2}
$$

The other representation of $R$ in the space-like region can be obtained by analytically continuing $\Im \Pi(s)$ from the time-like to the space-like region by means of a twice subtracted dispersion relation. This finally gives

$$
R^{\mathrm{HAD}}\left(M^{2}\right)=\frac{\Re \Pi^{\mathrm{HAD}}(0)}{M^{2}}-\frac{1}{\pi M^{2}} \int_{0}^{\infty} d s \Im \Pi^{\mathrm{HAD}}(s) \frac{s}{s^{2}+\epsilon^{2}} \exp -s / M^{2}
$$


Here $\Pi^{\mathrm{HAD}}$ represents a phenomenological hadronic spectral function. Since for the vector mesons this spectral function is dominated by resonances in the low-energy part it is usually parametrized in terms of a resonance part with parameters such as strength, mass and width at low energies with a connection to the QCD perturbative result for the quark structure for the current-current correlator at higher energies (for details see the contribution by S. Leupold et al. \& in these proceedings and refs. [9, 10, 11]).

The QCD sum rule is then obtained by setting

$$
R^{\mathrm{OPE}}\left(M^{2}\right)=R^{\mathrm{HAD}}\left(M^{2}\right)
$$

Knowing the lhs of this equation then allows one to determine the parameters in the spectral function appearing in $R^{\mathrm{HAD}}$ on the rhs. If the vector meson moves in the nuclear medium, then $R$ depends also on its momentum. However, detailed studies [10, 11] find only a very weak momentum dependence.

The first applications [1] of the QCDSR have used a simplified spectral function, represented by a $\delta$-function at the meson mass and a perturbative QCD continuum, starting at about $s \approx 1.2 \mathrm{GeV}$. Such an analysis gives a value for the free meson mass that agrees with experiment. On this basis the QCDSR has been applied to the prediction of in-medium masses of vector mesons by making the condensates density-dependent (for details see [1, 9, 10]). This then leads to a lowering of the vector meson mass in nuclear matter.

This analysis has recently been repeated with a spectral function that uses a Breit-Wigner parametrization with finite width. In this study [9] it turns out that QCD sum rules are compatible with a wide range of masses and widths (see also ref. [7]). Only if the width is - artificially kept zero, then the mass of the vector meson has to drop with nuclear density [四]. However, also the opposite scenario, i.e. a significant broadening of the meson at nearly constant pole position, is compatible with the QCDSR.

\subsection{Hadronic models}

Hadronic models for the in-medium properties of hadrons start from known interactions between the hadrons and the nucleons. In principle, these then allow one to calculate the forward scattering amplitude $f_{\mathrm{VN}}$ for vector meson interactions, for example. Many such models have been developed over the last few years [7, 12, 13, 14, 15].

The model of Friman and Pirner [14] was taken up by Peters et al. [16] who also included $s$-wave nucleon resonances. It turned out that in this analysis the $D_{13} N(1520)$ resonance plays an overwhelming role. This resonance has a significant $\rho$ decay branch of about $20 \%$. Since at the pole energy of $1520 \mathrm{MeV}$ the $\rho$ decay channel is not yet energetically open this decay can only take place through the tails of the mass distributions of resonance and meson. The relatively large relative decay 


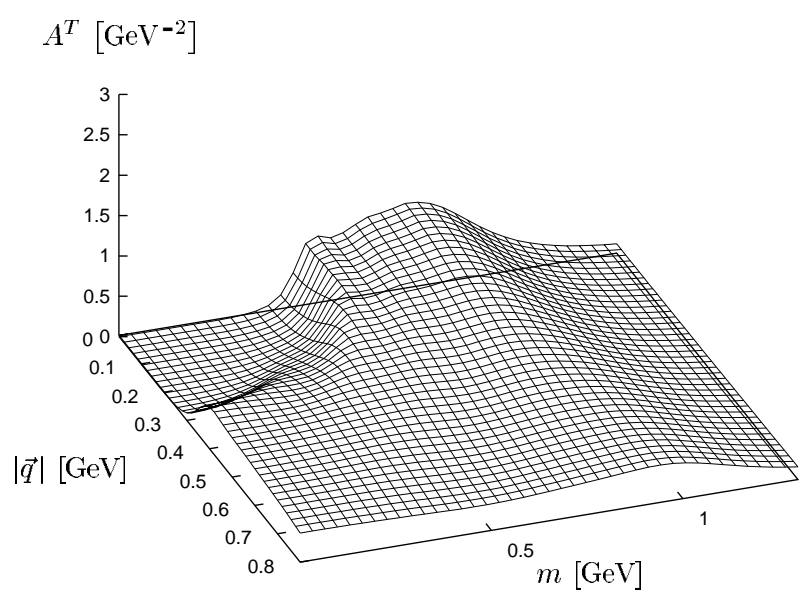

Figure 1: Spectral function of transverse $\rho$ mesons as a function of their three-momentum and invariant mass (from [16]).

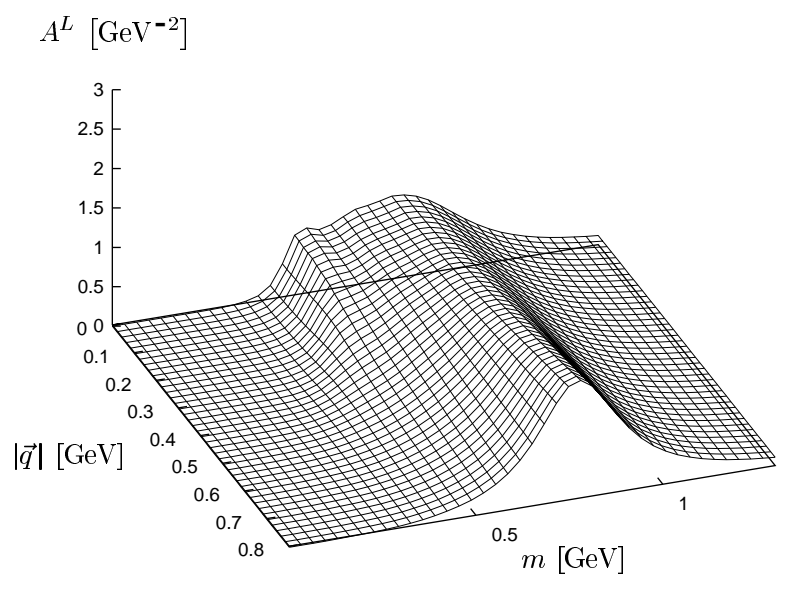

Figure 2: Spectral function of longitudinal $\rho$ mesons as a function of their three-momentum and invariant mass (from [16]).

branch then translates into a very strong $N^{*} N \rho$ coupling constant (see also [17, 15]).

The main result of this $N^{*} h$ model for the $\rho$ spectral function is a considerable broadening for the latter. This is primarily so for the transverse vector mesons (see Fig. 国), whereas the longitudinal degree of freedom gets only a little broader with only a slight change of strength downwards (see Fig. 2[16].

The results shown in Fig. 1 1 actually go beyond the simple " $t \rho "$ approximation discussed earlier (see (1) ) in that they contain higher order density effects: a lowering of the $\rho$ meson strength leads to a strong increase of the phase-space available for decay of the $N(1520)$ resonance; this causes a corresponding strong increase of the $N(1520) \rho$-decay width which in turn affects the spectral function. The result is the very broad, rather featureless spectral function for the transverse $\rho$ shown in Fig. 11. 

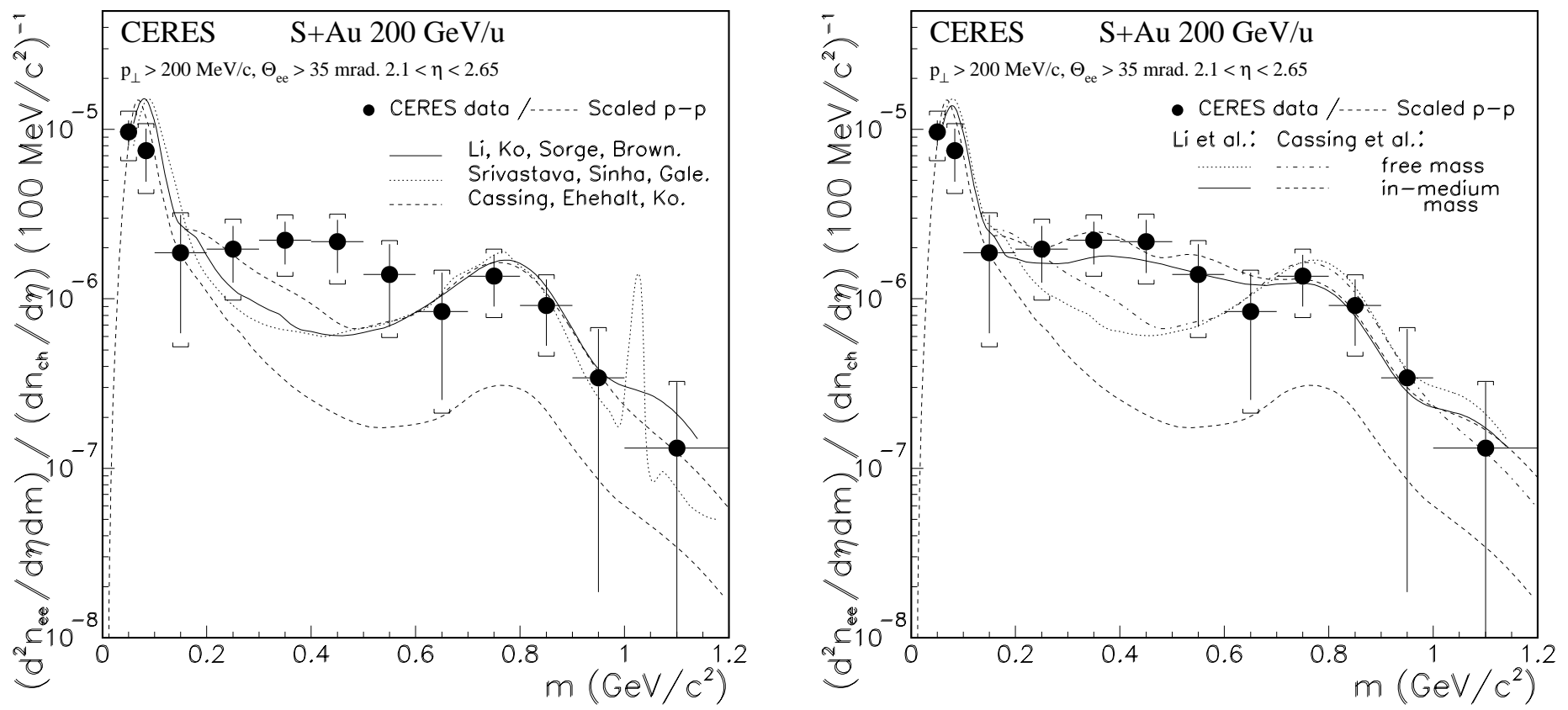

Figure 3: Comparison of several transporttheoretical calculations of the dilepton invariant mass spectrum with the CERES data. The calculations on the left show results of calculations employing free hadron properties, those on the right employ in-medium corrections to the $\rho$ meson (solid and dashed lines) (from [22]).

\section{Experimental Observables}

In this section I will now discuss various possibilities to verify experimentally the predicted changes of the $\rho$ meson properties in medium.

\subsection{Heavy-Ion Reactions}

Early work [18, 19] on an experimental verification of the predicted broadening of the $\rho$ meson spectral function has concentrated on the dilepton spectra measured at relativistic energies (about $1-4 \mathrm{~A}$ $\mathrm{GeV}$ ) at the BEVALAC, whereas more recently many analyses have been performed for the CERES and HELIOS data obtained at ultrarelativistic energies $(150-200 \mathrm{~A} \mathrm{GeV})$ at the SPS. In such collisions nuclear densities of about $2-3 \rho_{0}$ can already be reached in the relativistic domain; in the ultrarelativistic energy range baryon densities of up to $10 \rho_{0}$ are predicted (for a recent review see [20]). Since the selfenergies of produced vector mesons are at least proportional to the density $\rho$ (see (11)) heavy-ion reactions seem to offer a natural enhancement factor for any in-medium changes.

The CERES data 21] indeed seem to confirm this expectation (see Fig. 3). The left calculation shows that a large part of the CERES dilepton yield can already be explained by simple secondary reactions, mainly by $\pi^{+} \pi^{-} \rightarrow e^{+} e^{-}$because of the known high pion multiplicity in such high-energy heavy-ion reactions. Even then, there is, however, some yield still missing. The present situation is 
- independent of the special model used for the description of the data - that agreement with the measured dilepton mass spectrum in the mass range between about 300 and $700 \mathrm{MeV}$ for the $200 \mathrm{~A}$ $\mathrm{GeV} S+A u$ and $S+W$ reactions can only be obtained if $\rho$-meson strength is shifted downwards (for a more detailed discussion see [20, 23]) (for the recently measured $158 \mathrm{~A} \mathrm{GeV} \mathrm{Pb}+\mathrm{Au}$ reaction the situation is not so clear; here the calculations employing 'free' hadron properties lie at the lower end of the experimental error bars [20]).

However, all the predictions are based on equilibrium models in which the properties of a $\rho$ meson embedded in nuclear matter with infinite space-time extensions are calculated. A ultrarelativistic heavy-ion collision is certainly far away from this idealized scenario. In addition, heavy-ion collisions necessarily average over the polarization degrees of freedom. The two physically quite different scenarios, broadening the spectral function or shifting simply the $\rho$ meson mass downwards while keeping its free width, thus lead to indistinguishable observable consequences in such collisions. This can be understood by observing that even in an ultrarelativistic heavy-ion collision, in which very high baryonic densities are reached, a large part of the observed dileptons is actually produced at rather low densities (see Fig. 3 in 24]).

\section{$4 \pi+A$ Reactions}

Motivated by this observation we have performed calculations of the dilepton invariant mass spectra in $\pi^{-}$induced reactions on nuclei [25]; the experimental study of such reactions will be possible in the near future at GSI. The calculations are based on a semiclassical transport theory, the so-called Coupled Channel BUU method (for details see [26]) in which the nucleons, their resonances up to 2 $\mathrm{GeV}$ mass and the relevant mesons are propagated from the initial contact of projectile and target until the final stage of the collision. This method allows one to describe strongly-coupled, inclusive processes without any a-priori assumption on the equilibrium or preequilibrium nature of the process. Results of these calculations are being published in [25].

Since then, we have improved the calculations in several aspects (see also [27]). First, we now include also processes like $N^{*} \rightarrow \Delta \rho$ in the $\rho$ production channel. We find that this channel yields sizeable contributions to the low-mass $\rho$ yield coming from the decay of rather high-lying $(\approx 1.9 \mathrm{GeV})$ nucleon resonances. Second, we now use a more sophisticated treatment of broad resonances (i.e. the $\rho$ ), that resembles that generally used for the treatment, e.g., of the $\Delta$ in transport calculations [33. Instead of producing the vector mesons only at their pole mass like in 25] and folding in the phasespace weighted spectral function, we now produce these particles already with the proper spectral function and thus propagate also mesons on the tail of the mass-distribution. This improvement, however, creates new problems: in these calculations very low mass $\rho$ and $\omega$ mesons sometimes reach 


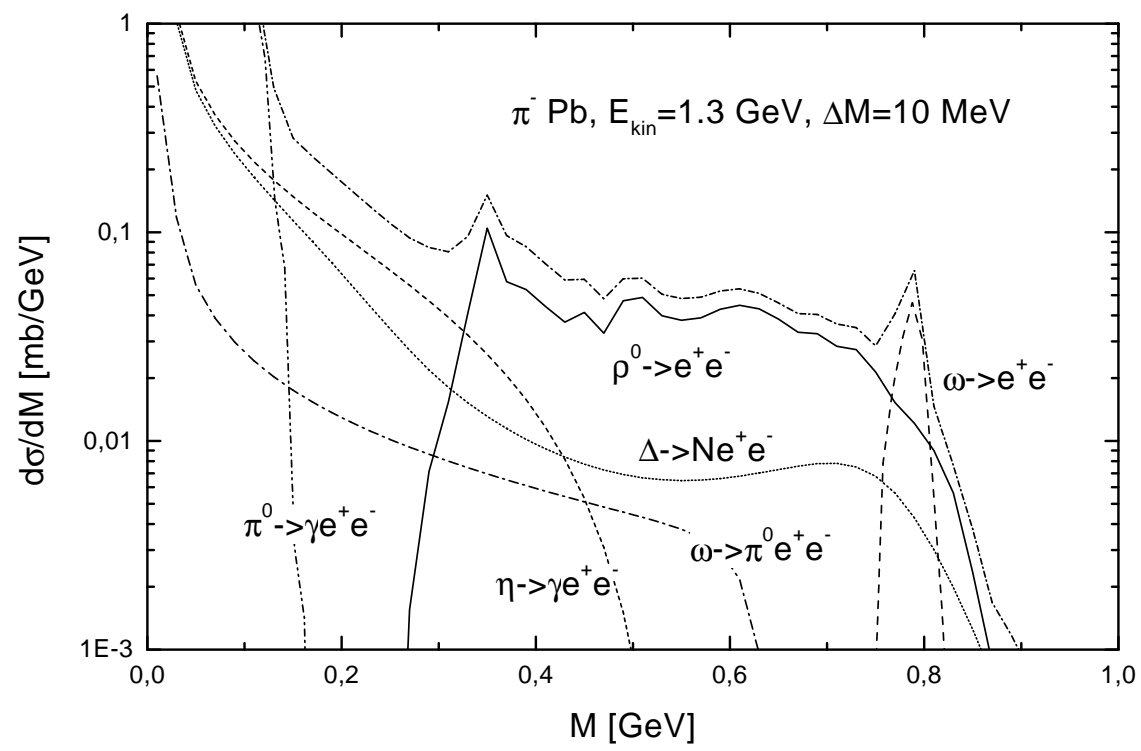

Figure 4: Invariant mass yield of dileptons produced in pion-induced reactions at $1.3 \mathrm{GeV}$ on $\mathrm{Pb}$ (from [29]).

the nuclear surface and escape, thus leading to a peak in the dilepton spectrum at very low masses. (the structures in Fig. 1 below the vector meson mass are due to statistical fluctuations and not to this effect). We cure this problem at present only heuristically by including a density-dependent selfenergy that ensures that all physical particles freeze out with their vacuum spectral distribution. And finally, we now use a VMD mandated $1 / M^{3}$ weighting for the dilepton spectra, which is consistent for calculations employing free hadron properties.

In these reactions the dominant dilepton emission channels are the same as in ultrarelativistic heavy-ion collisions; this can be seen in Fig. Ð where I show the results for the dilepton spectra produced by bombarding $\mathrm{Pb}$ nuclei with $1.3 \mathrm{GeV}$ pions. Up to about $400 \mathrm{MeV}$ invariant mass the strongest component is given by the $\eta$ Dalitz decay where the $\eta$ 's are produced through the experimentally rather well known process $\pi N \rightarrow \eta N$. Very close in magnitude in this lower mass range is the somewhat model-dependent $\pi N$ bremsstrahlung component (not shown here). In the vector meson mass range primarily both $\rho$ and $\omega$ contribute to the dilepton yield. First, still preliminary calculations indicate that the in-medium changes in the dilepton mass spectrum are of the order of a factor 2 in the mass range between $300 \mathrm{MeV}$ and $700 \mathrm{MeV}$ [29].

There are various interesting problems in this process that ultimately have to be answered by experiment. First, the $\pi N$ bremsstrahlung is quite uncertain. Parts of it are included in a calculation that includes processes like $\pi+N \rightarrow N^{*} \rightarrow N e^{+} e^{-}$(s-channel contributions), but $t$-channel processes for $\pi N$ scattering are not so easy to handle, because the frequently used long-wavelength approximation is known to be quite unreliable [28]. An exclusive measurement of pion-induced dilepton emission from 
the proton would be highly desirable to investigate this point.

A further interesting problem is how to determine the branching ratios of vector mesons into the dilepton channel. Simple VMD relates the coupling strength to the bare (pole) mass $m_{\rho}$ of the meson so that one obtains $\Gamma_{\rho \rightarrow e^{+} e^{-}}(M) \sim 1 / M^{3}$. However, in medium the vector meson is strongly coupled, e.g. to $N^{*} h$ excitations, which may lead to mass-dependent vertex corrections.

\section{Photonuclear Reactions}

Pion induced reactions have the disadvantage that the projectile already experiences strong initial state interactions so that many produced vector mesons are located in the surface where the densities are low. A projectile that is free of this undesirable behavior is the photon.

\subsection{Dilepton Production}

We have therefore - also in view of a corresponding proposal for such an experiment at CEBAF [30] - performed calculations for the dilepton yield expected from $\gamma+A$ collisions.

Results of these calculations are shown in Fig. 5. In the top figure the various sources of dilepton radiation are shown. The dominant sources are again the same as those in pion- and heavy-ion induced reactions, but the (uncertain) $\pi N$ bremsstrahlung does not contribute in this reaction. The middle part of this figure shows both the Bethe-Heitler $(\mathrm{BH})$ contribution and the contribution from all the hadronic sources. In the lowest (dot-dashed) curve we have chosen a cut on the product of the fourmomenta of incoming photon $(k)$ and lepton $(p)$ in order to surpress the BH contribution. It is seen that even without BH subtraction the vector meson signal surpasses that of the BH process.

The lowest figure, finally, shows the expected in-medium effects [31]: the sensitivity in the region between about 300 and $700 \mathrm{MeV}$ amounts to a factor of about 3 and is thus in the same order of magnitude as in the ultrarelativistic heavy-ion collisions.

In addition, the calculated strong differences in the in-medium properties of longitudinal and transverse vector mesons can probably only be verified in photon-induced reactions, where the incoming polarization can be controlled; this is true also for dilepton production with virtual, spacelike photons from inelastic electron scattering. Another approach would be to measure the coherent photoproduction of vector mesons; here the first calculation available so far [17] shows a distinct difference in the production cross sections of transverse and longitudinal vector mesons.

\subsection{Photoabsorption}

Earlier in this paper I have discussed that a strong change of the $\rho$ meson properties comes about because of its coupling to $N^{*} h$ excitations and that this coupling - through a higher-order effect - in 


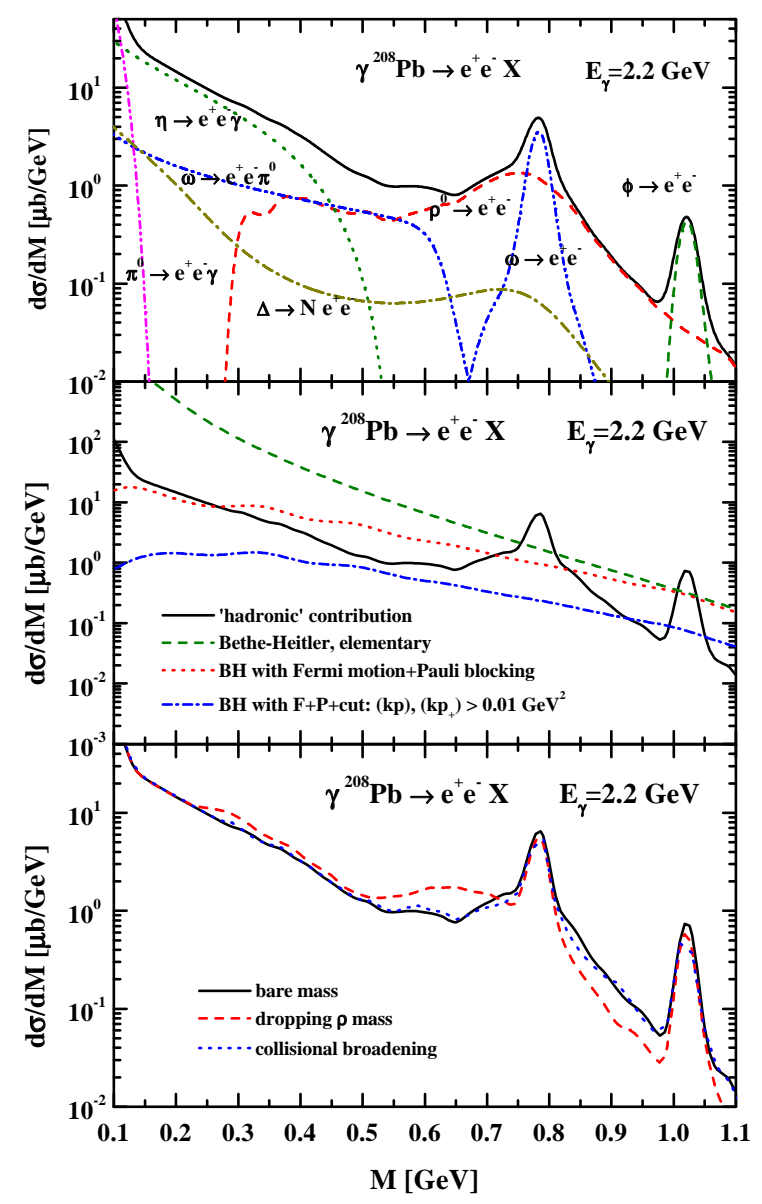

Figure 5: Invariant mass spectra of dileptons produced in $\gamma+{ }^{208} \mathrm{~Pb}$ reactions at $2.2 \mathrm{GeV}$. The top figures shows the various radiation sources, the middle figure the total yield from the top together with the Bethe-Heitler contributions, and the bottom part shows the expected in-medium effects (from [31). 


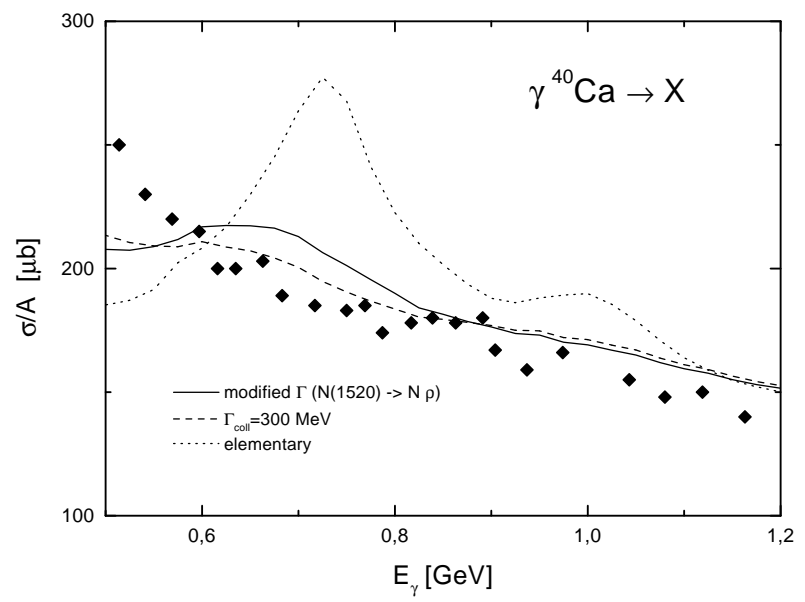

Figure 6: Photoabsorption cross section in the second resonance region. Shown are the data from ref. 32], the free absorption cross section on the proton, a Breit-Wigner fit with a total width of 300 $\mathrm{MeV}$ (dashed curve) and the result of a transporttheoretical calculation 33] with a medium broadened $\rho$ decay width of the $N(1520)$ (solid curve).

particular leads to a very strong increase of the $\rho$ decay width of the $N(1520) D_{13}$ resonance.

This increase may provide a reason for the observed disappearance of the higher nucleon resonances in the photoabsorption cross sections on nuclei [32. These cross sections scale very well with the massnumber $A$ of the nucleus, which indicates a single-nucleon phenomenon. They also clearly show a complete absence of the resonance structure in the second and third resonance region. The disappearance in the third region is easily explainable as an effect of the Fermi-motion. The disapparance of the second resonance region, i.e. in particular of the $N(1520)$ resonance, however, presents an interesting problem; it is obviously a typical in-medium effect.

First explanations [4] assumed a very strong collisional broadening, but in ref. 33 it has been shown that this broadening is not strong enough to explain the observed disappearance of the $D_{13}$ resonance. Since at the energy around $1500 \mathrm{MeV}$ also the $2 \pi$ channel opens it is natural to look for a possible connection with the $\rho$ properties in medium. Fig.6 shows the results of such an analysis (see also [17]). It is clear that the opening of the phase space for $\rho$ decay of this resonance provides enough broadening to explain its disappearance.

\section{Summary}

In this talk I have concentrated on a discussion of the in-medium properties of the $\rho$ meson. I have shown that the scattering amplitudes of the $\rho$ meson on nucleons determine the in-medium spectral function of the $\rho$, at least in lowest order in the nuclear density. The dilepton channel can give 
information on the properties of the $\rho$ deep inside nuclear matter whereas the $2 \pi$ decay channel because of its strong final state interaction - can give only information about the vector meson in the low-density region.

The original QCD sum rule predictions of a lowered $\rho$ mass have turned out to be too naive, because they were based on the assumption of a sharp resonance state. In contrast, all specific hadronic models yield very broad spectral functions for the $\rho$ meson with a distinctly different behavior of longitudinal and transvese $\rho$ 's. Recent QCD sum rule analyses indeed do not predict a lowering of the mass, but only yield - rather wide - constraints on the mass and width of the vector mesons. I have also discussed that hadronic models that include the coupling of the $\rho$ meson to nucleon resonances and a corresponding shift of vector meson strength to lower masses give a natural backreaction on the width of these resonances. In particular, the $N(1520) D_{13}$ resonance is significantly broadened because of its very large coupling constant to the $\rho N$ channel. Since the $\rho$ decay of this resonance has never been directly seen in an experiment, but is deduced only from one partial wave analysis of $2 \pi$ production [36], it would be very essential to have new, better data (and a new analysis of these) for this channel.

A large part of the unexpected surplus of dileptons produced in ultrarelativistic heavy-ion collisions can be understood by simply including secondary interactions, in this case $\pi+\pi \rightarrow \rho$, in the 'cocktail plot' of hadronic sources; this special source is one that is particular to heavy-ion collisions with their very large pion multiplicity. Since the pions are produced rather late during the collision, the gain from the high densities reached in heavy ion collisions is not so large as one could naively have expected.

Motivated by this observation I have then discussed predictions for experiments using pion and photon beams as incoming particles. In both cases the in-medium sensitivity is nearly as large as it is in the heavy-ion experiments. In addition, such experiments have the great advantage that they take place much closer to equilibrium, an assumption on which all predictions of in-medium properties are based. Furthermore, only in such experiments it will be possible to look for polarization effects in the in-medium properties of the $\rho$ meson.

I have finally shown that the in-medium properties of the $\rho$ also show up in photonuclear reactions. One intriguing suggestion is that the observed disappearance of the second resonance region in the photoabsorption cross section is due to the broadening of the $N(1520)$ resonance caused by the shift of $\rho$ strength to lower masses.

At high energies, finally, the in-medium broadening of the $\rho$ leads to a mean free path of about $2 \mathrm{fm}$ in nuclear matter. Shadowing will thus only be essential if the coherence length is larger than this mean free path. Increasing the four-momentum transfer $Q^{2}$ at fixed energy transfer $\nu$ leads to smaller coherence length and thus diminishes the initial state interactions of the photon leading to 
a larger transparancy. This effect is essential to verify experimentally; it is superimposed on the color-transparency effect that is still being looked for.

\section{Acknowledgement}

This talk is based on results of work with E. Bratkovskaya, W. Cassing, M. Effenberger, H. Lenske, S. Leupold, W. Peters, M. Post and T. Weidmann. I am grateful to all of them for many stimulating discussions. I wish to thank in particular M. Effenberger and E. Bratkovskaya for providing me with the results shown in Figs. 4 and 5 before publication.

\section{References}

[1] T. Hatsuda and S.H. Lee, Phys. Rev. C 46 (1992) R34

[2] G.E. Brown and M. Rho, Phys. Rev. Lett. 66 (1991) 2720

[3] V.L. Eletsky and B.L. Ioffe, Phys. Rev. Lett. 78 (1997) 1010

[4] L.A. Kondratyuk, M.I. Krivoruchenko, N. Bianchi, E. De Sanctis and V. Muccifora, Nucl. Phys. A $579(1994) 453$

[5] J.J. Sakurai, Currents and Mesons, University of Chicago Press, Chicago (1969)

[6] M.E. Peskin and D.V. Schroeder, An Introduction to Quantum Field Theory, Addison-Wesley, Reading 1995

[7] F. Klingl and W. Weise, Nucl. Phys. A 606 (1996) 329

[8] S. Leupold and U. Mosel, contribution to this meeting

[9] S. Leupold, W. Peters, and U. Mosel, Nucl. Phys. A 628 (1998) 311

[10] S. Leupold and U. Mosel, Phys. Rev. C 58 (1998) 2939

[11] S.H. Lee, Phys. Rev. C 57 (1998) 927

[12] M. Hermann, B. Friman and W. Noerenberg, Nucl. Phys. A 545 (1992) 267c

[13] M. Asakawa and C.M. Ko, Phys. Rev. C 48 (1993) 526

[14] B. Friman and H.J. Pirner, Nucl. Phys. A 617 (1997) 496

[15] R. Rapp, G. Chanfray, and J. Wambach, Nucl. Phys. A 617 (1997) 472 
[16] W. Peters, M. Post, H. Lenske, S. Leupold, and U. Mosel Nucl. Phys. A 632 (1998) 109

[17] M. Effenberger, M. Post, and U. Mosel, contribution to BARYONS'98, in press

[18] G. Wolf, W. Cassing, W. Ehehalt, and U. Mosel, Progr. Part. Nucl. Phys. 30 (1993) 273

[19] L. Xiong, Z.G. Wu, C.M. Ko, and J.Q. Wu, Nucl. Phys. A 512 (1990) 772

[20] W. Cassing and E.L. Bratkovskaya, Phys. Rep., in press

[21] G. Agakichiev et al., PRL 75 (1995) 1272, Phys. Lett. B 422 (1998) 405

[22] C.P. Heros et al., preprint CERN-PPE/96-111, talk given at the XXX1st Rencontres de Morion on QCD and High Energy Hadronic Interactions, Les Arcs, 1996

[23] For a comprehensive review see Proc. Quark Matter 96, Nucl. Phys. A 610 (1996) 317c - 371c

[24] W. Cassing, W. Ehehalt, and I. Kralik, Phys. Lett. B 377 (1996) 5

[25] T. Weidmann, E. Bratkovskaya, W. Cassing and U. Mosel, Phys. Rev. C, in press

[26] S. Teis, W. Cassing, M. Effenberger, A. Hombach, U. Mosel, and Gy. Wolf, Z. Phys. A 356 (1997) 421

[27] J. Knoll, talk at this meeting

[28] P. Lichard, PRC 51 (1995) 6017

[29] M. Effenberger, to be published

[30] P. Bertin et al., CEBAF proposal E94002

[31] M. Effenberger, E. Bratkovskaya and U. Mosel, to be published

[32] N. Bianchi et al, Phys. Rev. C 54 (1996) 1688

[33] M. Effenberger, A. Hombach, S. Teis, and U. Mosel, Nucl. Phys. A 613 (1997) 353

[34] N. Bianchi, Proc. BARYONS'98, in press

[35] L.A. Kondratyuk, A. Sibirtsev, Ye.S. Golubeva, and M. Effenberger, Phys. Rev. C 58 (1998) 1078

[36] D.M. Manley and E.M. Saleski, Phys. Rev. D 54 (1992) 4002 